\title{
Temporally and Spatially Resolved Measurements in the Plume of Clustered Hall Thrusters
}

\author{
Robert B. Lobbia, ${ }^{*}$ Thomas M. Liu, ${ }^{\dagger}$ and Alec D. Gallimore ${ }^{*}$ \\ The University of Michigan, Ann Arbor, MI, 48109
}

\begin{abstract}
A High-speed Dual Langmuir Probe system (HDLP) and a high-speed camera tracked 19.6-kHz waves of ionized propellant emitted by the unsteady ionization process or "breathing mode" of a cluster of Hall thrusters. Two 600-W, low-power Hall thrusters were operated together in close proximity with 200-V, 2-A xenon discharges, producing the plume examined in this study. Large amplitude plasma oscillations, with peak-to-peak magnitudes approaching $100 \%$ of the DC level, are observed in the near- and far-field plume electron density, optical intensity, and thruster discharge current. The HDLP system acquired the plume electron density in a planar grid measuring $0.31 \mathrm{~m}$ radially, centered on cluster centerline (with 10-mm spacing), and $0.5 \mathrm{~m}$ axially downstream (with $50-\mathrm{mm}$ spacing). The structural evolution of the exhausted plasma density fluctuations was resolved for this entire grid at a rate of $100 \mathrm{kHz}$ (using methods of Fourier analysis with the HDLP data) while a high-speed camera imaged the two discharge channels at a rate of 67,500 frames-per-second (with the discharge currents of each thruster sampled at $2 \mathrm{MHz}$ ). Recorded synchronously, these independent spatially and temporally resolved measurements correlated well with each other and revealed the complex nature of the interactions between the two simultaneously discharging Hall thrusters. Interactions in the near-field plume proved viable enough to encourage coupled breathing mode oscillations, in which the thrusters preferentially breathed in an in-phase "mode-0" (observed $43.4 \%$ of the run duration) or an opposite-phase "mode-1" (36.1\%) manner. Comparisons of these coupled breathing mode oscillations observed in the clustered thrusters were made with single-thruster breathing mode oscillations. For both configurations, a non-dimensional, localized electron density mean fluctuation peak-topeak magnitude of about $72 \% \pm 10 \%$ was seen at all radial and axial plume locations.
\end{abstract}

\section{Introduction}

Therent to the ionization scheme employed in Hall effect thrusters (HETs), intense "breathing mode" oscillations of ejected plasma dominate the temporal progression while operating most Hall thrusters developed to date. Usually, these oscillations are measured in-line with the power supply driving the thruster discharge current, yet it has been shown that these large amplitude fluctuations exist in time-resolved measurements of the near- and farfield plume electron density, electron temperature, and plasma potential. ${ }^{1,2}$ In contrast to the conventionally labeled "electrostatic" description of HET operation, these unsteady transient breaths of plasma unveil a strong "dynamic" behavior with distinct ion waves, thermal waves, and electromagnetic waves propagating downstream from the thruster, relatively undamped, at distances in excess of 10 discharge channel diameters. The breathing mode also produces an unsteady emission of photons generated by increased atomic state transitions associated with the rapid bursts of ionization. Even with the considerable body of prior research regarding the breathing mode of Hall thrusters, ${ }^{3,4,5}$ the two-dimensional, time-resolved structure and evolution of the resultant plasma properties has been hitherto unknown. The demand for higher power Hall thruster propulsion based missions has led to the concept of clustering together arrays of Hall thrusters as a means for achieving large power levels that cannot be tested in conventional vacuum chambers ${ }^{6}$. While the DC effects of clustering have been investigated, ${ }^{7,8}$ detailed measurement of the unique transient behavior arising from arrays of clustered Hall thrusters is yet unknown. In this work, an assortment of time- and space-resolved measurements acquired simultaneously (discharge current, electron density, and photographic intensity) are compared and correlated with each other and between single thruster and clustered configurations.

Arising from the myriad of electromagnetic interactions between excited propellant particles, electrons, and the imposed magnetic and electric fields, HET plasmas are rich with oscillation in a wide band ranging from $1 \mathrm{kHz}$ to $20 \mathrm{GHz}^{3,9}$. However, the amplitude of the low-frequency breathing mode (typically $15-40 \mathrm{kHz}$ ) exceeds all other

\footnotetext{
*PhD Candidate, Aerospace Engineering Dept., FXB Room 1052, AIAA Student Member, lobbia@umich.edu.

$\dagger$ PhD Candidate, Aerospace Engineering Dept., FXB Room 1052, AIAA Student Member, liutm@umich.edu.

* Arthur F. Thurnau Prof., Aerospace Engineering Dept., Associate Fellow AIAA, alec.gallimore@umich.edu.
} 
modes by orders of magnitude, thereby warranting increased attention and the focus of this study. Understanding this dynamic nature of Hall thruster propulsion is critical for a variety of reasons:

- Insight into the performance and lifetime effects of the breathing mode oscillations may allow for improved Hall thruster designs that either amplify or attenuate the oscillations.

- Fluctuations in the plume plasma potential create electromagnetic interference emissions that could adversely affect spacecraft communication, adjacent thrusters, or other sensitive spacecraft electronics.

- $\quad$ The clustering of Hall thrusters in close proximity may cause adverse interactions between adjacent thrusters - potentially shortening lifetime or reducing performance.

Addressing these topics requires a better understanding of HET dynamical behavior in both clustered and single thruster configurations.

\section{Experimental Setup}

\section{A. Large Vacuum Test Facility and 4x 600-W HET Cluster}

Testing was performed in the cylindrical (6-m diameter and 9-m length), stainless-steel-clad Large Vacuum Test Facility (LVTF) at the University of Michigan. Running four of seven cryogenic pumps, an aggregate pumping rate of $120 \mathrm{kl} / \mathrm{s}$ on xenon maintains a high-vacuum pressure of $1 \times 10^{-5}$ Torr during single thruster operation, and $1.8 \times 10^{-5}$ Torr with two thruster clustered operation (corrected for xenon). The 2-by-2 cluster of four low-power, 600-W Hall thrusters utilized in this experiment is shown, as mounted, in Figure 1. The four thrusters (each with its own cathode) are labeled in a counter-clockwise manner beginning with the upper-left Thruster1 or T1. Presented data include single thruster firing of $\mathrm{T} 3$, as well as two thruster firing of $\mathrm{T} 2$ and T3. The data collection region exists in the plane intersecting these lower two thrusters, spanning $31 \mathrm{~cm}$ radially about their axis of symmetry and extending from 10 $\mathrm{cm}$ to $60 \mathrm{~cm}$ axially downstream from the thruster exit plane. A dedicated set of heater, keeper, inner and outer

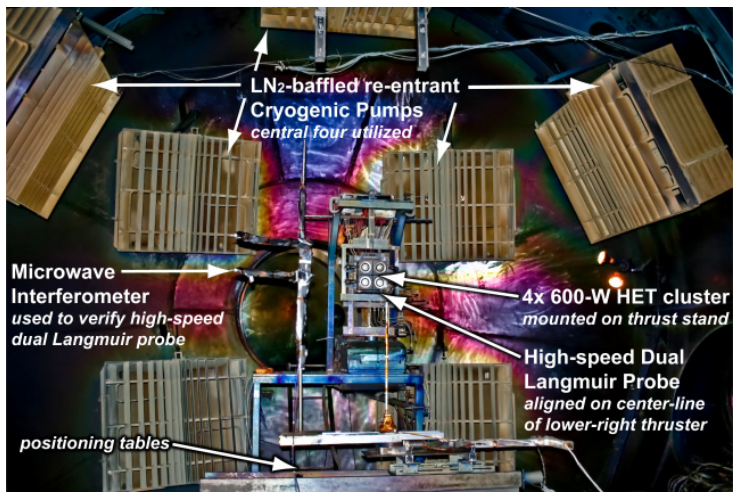

Figure 1. Two-by-two 600-W Hall thruster cluster mounted inside LVTF. The $100-\mathrm{kHz}$ dual Langmuir probe (HDLP) is positioned $10 \mathrm{~cm}$ downstream from Thruster 3 (T3), the lower-right thruster (the lower-left is T2). electromagnet, and discharge power supplies (unfiltered), along with a dedicated pair of anode and cathode mass flow controllers, are connected to each of the four thrusters in the cluster. The collection of data presented here were obtained after complete conditioning of the cathodes, as well as the anodes, to eliminate potential water vapor out-gassing ${ }^{10}$ that can cause additional dynamics during the first hour of thruster operation after atmospheric exposure. While each of the four thrusters are identical in construction and setup, the cumulative run hours of each thruster are different, giving rise to small differences in the discharge characteristics among the thrusters (e.g., a $2.2 \%$ difference in mean discharge current between $\mathrm{T} 2$ and $\mathrm{T} 3$ for the same $200-\mathrm{V}$ anode potential, electromagnet, and flow settings). Discharge current or thrust differences are usually removable by adjusting the individual thruster and cathode settings, but here no such efforts are made.

\section{B. High-speed Dual Langmuir Probe (HDLP) and High-speed Imaging}

The HDLP diagnostic is described well elsewhere, ${ }^{1}$ so only a short description is given in this work. Comprised of a traditional single Langmuir probe in close proximity to a capacitance compensating Null probe (insulated from the plasma), the HDLP system enables bias sweep rates in excess of $1 \mathrm{MHz}$. The rapidly acquired probe full current-voltage (I-V) characteristics are collected back-to-back (using a symmetric triangle bias signal), thereby (after conventional thin-sheath Langmuir probe analysis) providing seamless temporal records of electron density $\left(n_{e}\right)$, electron temperature $\left(T_{e}\right)$, and plasma potential $\left(V_{p}\right)$. For the presented data, an 100-kHz bias sweep rate was used, and at each spatial grid-point (352 altogether), 1 second of the following properties were simultaneously sampled: $n_{e}(x, y, t), T_{e}(x, y, t), V_{p}(x, y, t), I_{d 3}(t), I_{d 2}(t), F_{T 3}(t)$, and $F_{T 2}(t)$. The latter four signals of thruster discharge current $\left(I_{d}\right)$ and photographs (with normalized frame-integrated image intensity $F$ ) taken by a FASTCAM highspeed camera are sampled synchronously but at faster rates of $2 \mathrm{MHz}$ for current and 67,500 FASTCAM frames/sec. A pair of radial and axial positioning tables move the probe to each grid-point in sequence. A series of networked 
computers automate the data collection, which requires about 3 hours and $11 \mathrm{~GB}$ to map the $31-\mathrm{cm}$ by $50-\mathrm{cm}$ planar region with $10-\mathrm{mm}$ radial, $50-\mathrm{mm}$ axial, and $\leq 10-\mu$ s temporal intervals.

\section{Results}

A rather complex series of signal processing steps are used to fuse all the spatially distinct signals into a single two-dimensional, time-resolved grid and the reader is advised to see a prior publication that discusses this method in depth. ${ }^{11}$ In summary, matrices of transfer functions are used to model the turbulent statistics of each thruster with the plume in frequency space. Then, a single pair of inputs (FASTCAM-synced $I_{d 2}$ and $I_{d 3}$ measured signals) are used to synthesize the entire plume response. As the clustered thrusters are powered with independent power supplies and flow lines, the two matrix systems are accordingly treated as linearly independent from one another, thus allowing the principle of super-positioning to be applied in combining the two plume responses (one from each thruster). One additional step was taken to attain an estimate of the lower thruster transfer function model by reflection of matrix terms; this is not normally needed but was required here due to a shortage of DAQ channels in this experiment.

\section{Single Point Measurements}

Using this spatial-temporal data-fusion technique, one may then examine the two-dimensional plume region on a fixed set of temporally aligned intervals. For example in Figure 1, the plasma oscillations observed at two radial locations $(\mathrm{R}=9 \mathrm{~cm}, \mathrm{R}=0 \mathrm{~cm})$ for a fixed sequence of thruster discharge currents $(2 \mathrm{x}$ cluster mode with $200-\mathrm{V}$ and 2 A HET settings). The distinct $19.6 \mathrm{-kHz}$ breathing mode type oscillations observed in Figure 1(b) are similar to oscillations seen when running this thruster unclustered. ${ }^{1}$ Since this position is separated radially from the axis of the neighboring thruster by over $10 \mathrm{~cm}$, less influence is indeed expected. However, on cluster centerline as in Figure 1(c), nearly equivalent fluctuation contributions are provided by each breathing HET, thus creating less distinct plasma oscillations. The sum of the two thruster discharge current is included since the measured density transients appear to follow this effective signal (as expected from clustered HET plume density super-positioning ${ }^{7}$ ).
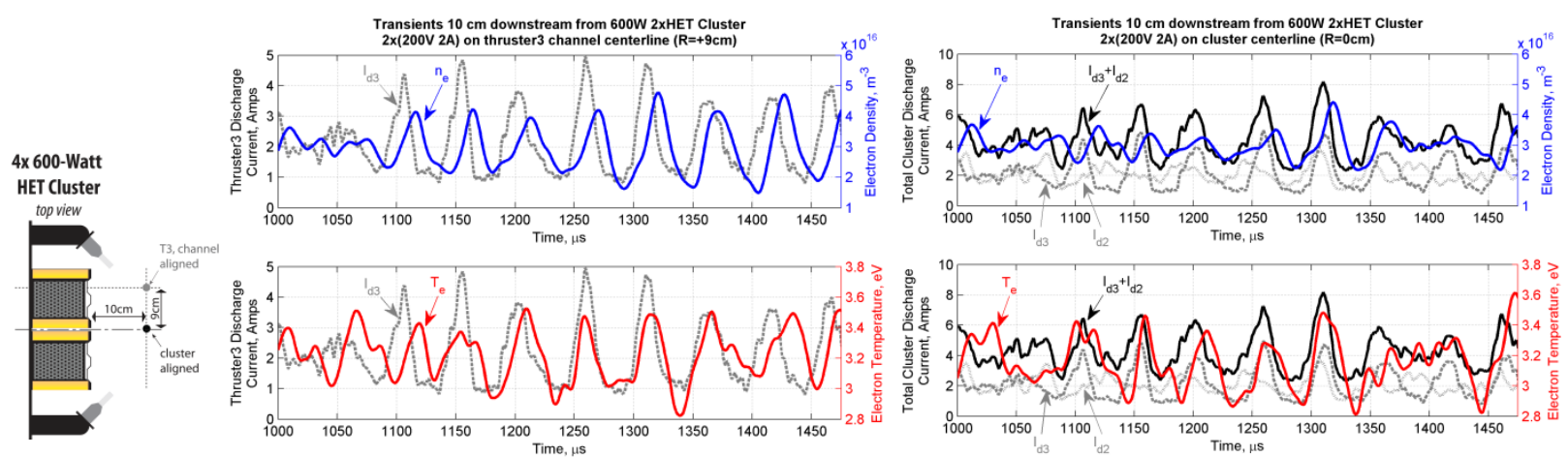

(a)

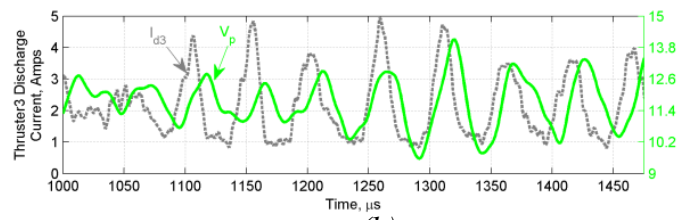

(b)

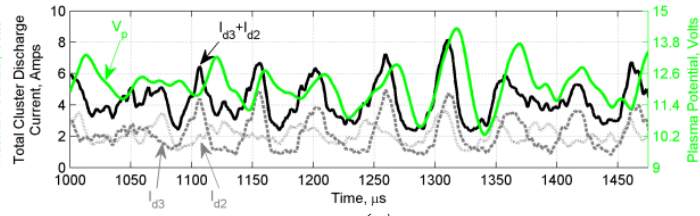

(c)

Figure 2. Single-point, simultaneous plume electron density, electron temperature, plasma potential, and clustered HET discharge currents for two radial positions. (a) Locations of the measurements relative to the cluster. (b) T3 discharge channel aligned (outer edge, away from T2) plume properties. (c) Cluster aligned properties including both separate thruster anode currents ( $I_{d 3}$ and $I_{d 2}$, gray dashed) and superimposed current $\left(I_{d 3}+I_{d 2}\right.$, solid black).

For electron density (equivalent to ion density with quasineutrality), the small phase lag $(\approx 10 \mu \mathrm{s})$ with respect to the discharge current corresponds to the ion transit time from the region of ionization to this location $10 \mathrm{~cm}$ downstream from the exhaust plane. A theoretical estimation of this time follows from: 


$$
\tau_{i, t}(x) \approx\left(x+L_{a}\right) \sqrt{\frac{m_{i}}{2 e \eta_{v} V_{d}}}, x \geq L_{a}
$$

with parameters of: ion mass $m_{i}$, constant-acceleration-zone length $L_{a}$, electron charge $e$, voltage utilization efficiency $\eta_{v}$, discharge voltage $V_{d}$, and distance from the ionization zone $x$. Setting $L_{a}$ as the discharge channel diameter $(\approx 5.5 \mathrm{~cm})$ and $\eta_{v}$ to $80 \%$, a theoretical ion transit time of $\tau_{i, t}(10 \mathrm{~cm}) \approx 10.1 \mu \mathrm{s}$ is predicted. Even with the simplicity of (1) this estimate agrees well with the measured $10 \mu$ s delay in Figure 1. A more statistically accurate measure of the phase between $I_{d 3}(t)$ and $n_{e}(t)$ is readily available by using a Bode plot of the transfer function at this location to determine the phase as $67.73^{\circ}$ at the breathing mode frequency: $\tau_{i, t x}(10 \mathrm{~cm})=\frac{67.73^{\circ}}{360^{\circ}} \cdot \frac{1}{19640 \mathrm{~Hz}}=9.6 \mu \mathrm{s} \pm 0.2 \mu \mathrm{s}$. The uncertainty is taken as the $95 \%$ confidence interval of the empirical transfer function.

\section{Clustered Modal Behavior}

Examination of the time-series signals suggests that the thrusters seem to prefer oscillating at the breathing mode in-phase with each other in what shall be defined as Mode- 0 . To quantify this behavior, the instantaneous phase between $I_{d 3}(t)$ and $I_{d 2}(t)$ is determined for a large set of samples. Using the same approach applied to compute $\tau_{i, t x}$, this is performed efficiently in frequency-space, where the phase between the signals at the breathing mode is simply the angle between the real and imaginary terms of the transfer function. The stochastic form of this phase encourages a statistical treatment. Thus, the probability density function (PDF) for all possible phases (at the breathing mode) is constructed by binning all phase states as in Figure 3. If the clustered thruster oscillations were entirely uncorrelated to one another, then a normal PDF (flat between $-180^{\circ}$ and $+180^{\circ}$ ) would be observed. However, two preferred modes of oscillation are observed: Mode- 0 with 43.4\% integrated probability and Mode- 1 (opposite phase oscillations) with $36.1 \%$ probability. The remaining $20.5 \%$ of oscillations appear at phases between Mode- 0 and Mode- 1 suggesting brief intermodal transitions. The predominance of Mode- 0 breathing mode oscillations produces more intense plasma waves as the thrusters breathe in-phase.

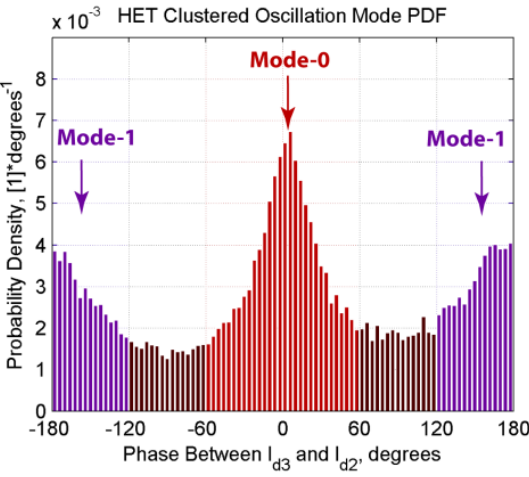

Figure 3. Probability density for measured phase between clustered thruster oscillations. Predominate with $43.4 \%$ integrated probability is Mode-0, with clustered thrusters oscillating in-phase. The close thruster positioning in the cluster tested is the likely reason for increased Mode- 0 oscillations since the thruster plumes possess considerable overlap. In particular, overlap in the external edges of the acceleration zones would surely give rise to coupled thruster oscillations due to the key role of the acceleration zone in breathing mode models. ${ }^{4}$ The Hall thrusters in this cluster are as close to each other as is physically allowed by the encasing outer electromagnets, thus the figure of $43.4 \%$ Mode- 0 behavior is likely an upper limit for clustered HETs. On real spacecraft, the thrusters would likely have additional spacing to alleviate thermal and vibration (launch) interactions. While the clustered experiments here were only performed with two thrusters, additional thrusters would increase the number of possible modes. Preliminary measurements with operating all four (2x2) HETs confirm this with the appearance of four dominant modes (Mode-0 all in-phase, Mode-1 right or left HETs in-phase, Mode-2 upper or lower HETs in-phase, and Mode-3 diagonal HETs in-phase).

\section{E. Planar Maps of Time-Averaged and Fluctuation Amplitude Electron Density}

The collected time-resolved planar electron density contour maps of clustered HET operation enable dramatic frame-after-frame animations, but the stochastic nature of the visualized breathing mode oscillations is best quantified by maps of the mean statistics. Thus, in Figure 4, the time-averaged electron density and mean fluctuation peak-to-peak amplitude planar contour maps are presented: 


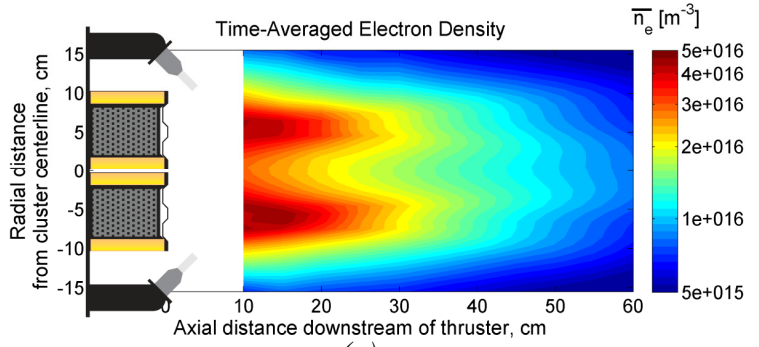

(a)

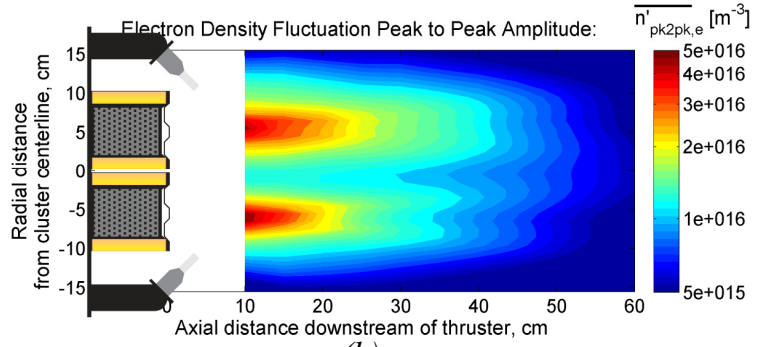

(b)

Figure 4. Electron density contour maps of (a) time-averaged and (b) fluctuation peak-to-peak amplitude values. Both (a) and (b) are plotted on the same logarithmic colorbar scale, emphasizing the large magnitudes of the emitted density waves relative to the local DC electron density values.

Slight differences in the thrusters lead to a small degree of asymmetry in the time-averaged and mean fluctuation amplitude maps. As was seen with single thruster operation, ${ }^{1}$ the mean peak-to-peak electron density fluctuation amplitudes nearly match the time-averaged electron densities at the same plume positions. Calculating the spatially averaged ratio of the fluctuation mean over temporal mean yields:

$$
\left.\left\langle\overline{\frac{n_{e}{ }^{\prime}{ }_{p k 2 p k}}{\overline{n_{e}}}}\right\rangle\right|_{\text {plume }(31 \mathrm{~cm} \text { by } 50 \mathrm{~cm})}=0.72
$$

This "non-dimensional fluctuation magnitude" varies only by about \pm 0.1 throughout the entire $31-\mathrm{cm}$ by 50 -cm plume region investigated with clustered operation. A similar, non-dimensional fluctuation amplitude was observed with single thruster operation. With both cases, the relative amplitude of the breathing mode oscillations remains constant (to within about 10\%) throughout the entire plume region studied. This implies the undamped persistence of breathing mode induced ion waves in all space downstream (with line of sight) from a HET until complete plume neutralization is reached.
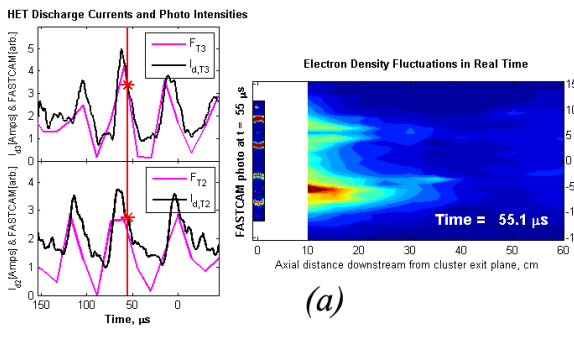

(a)
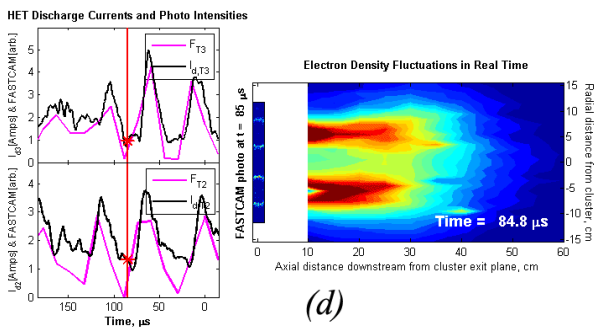

(d)
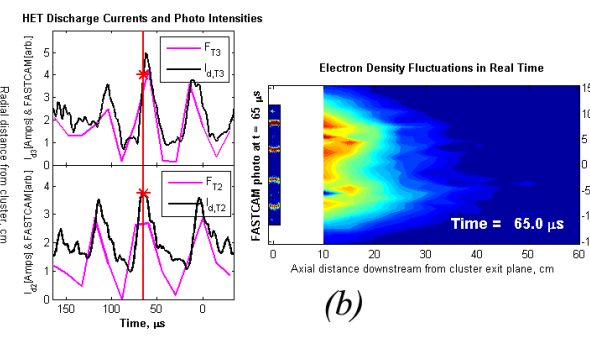

(b)
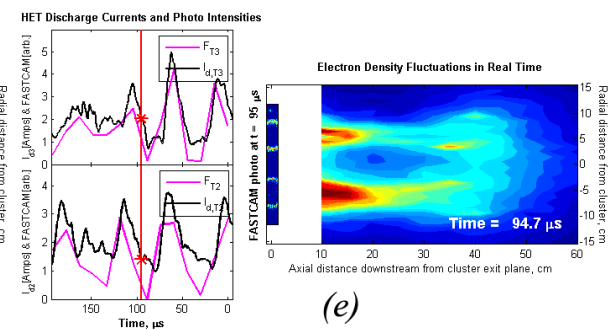

(e)
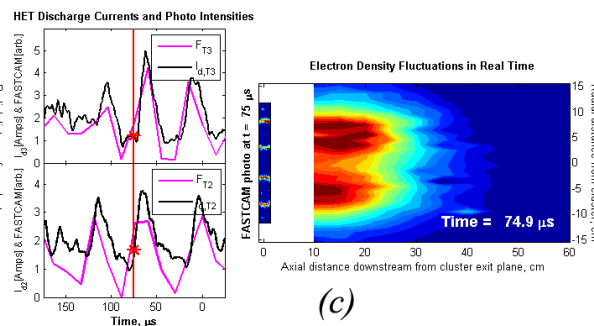

(c)
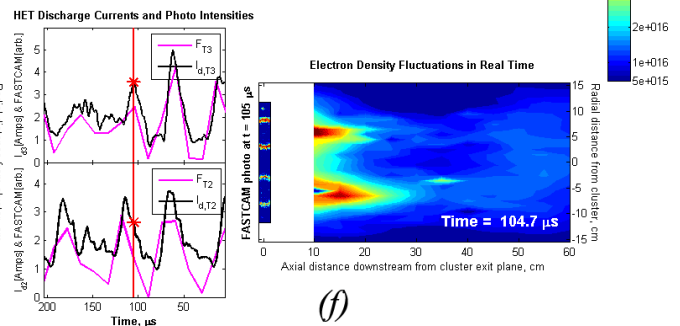

Figure 5. Temporally and spatially resolved electron density contours for one (mode-0 type) breathing mode sequence. The simultaneous multi-rate signals of discharge current (sampled at $2 \mathrm{MHz}$ ), photo intensity $(67.5 \mathrm{kHz})$, and electron density $(100 \mathrm{kHz})$ are included for each time interval: (a) $t=55.1 \mu \mathrm{s}$, (b) $t=65.0 \mu \mathrm{s}$, (c) $t=74.9 \mu \mathrm{s}$, (d) $t=84.8 \mu \mathrm{s}$, (e) $t=94.7 \mu \mathrm{s}$, and (f) $t=104.7 \mu \mathrm{s}$. 


\section{F. Temporally and Spatially Resolved Electron Density Results}

Figure 5 examines full planar plume density contour maps in a time-resolved sequence of frames that capture a complete mode- 0 breathing mode cycle. The discharge current signals for each thruster (T3 is upper and T2 is lower) are included to the left of each time-stamped contour map with a red asterisk marking these simultaneous

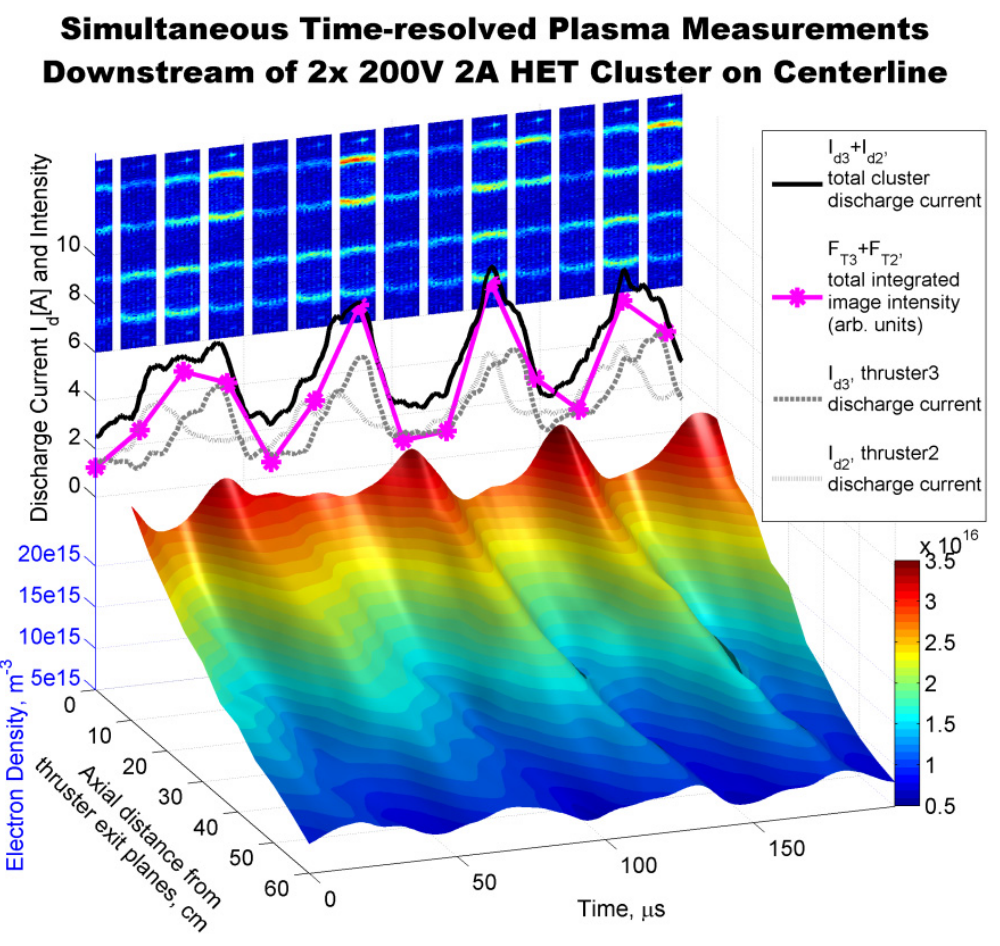

Figure 6. 3D axial electron density wave profiles on cluster centerline $(\mathbf{R}=\mathbf{0})$. The vertical axis combines all properties including: the time-resolved centerline electron density in blue (also colorbar scaled), separate thruster discharge currents $\left(I_{d 3}\right.$ and $\left.I_{d 2}\right)$, summed discharge currents $\left(I_{d 3}+I_{d 2}\right)$, total integrated intensity $\left(F_{T 3}\right.$ $\left.+F_{T 2}\right)$, and the time-aligned FASTCAM photos. current signals. The short delay (about $10 \mu \mathrm{s}$ ) between a burst of current and arrival of this transient at the plume map edge arises from propellant ion transit at an average velocity of $10.4 \mathrm{~km} / \mathrm{sec}$. Simultaneous FASTCAM photos are included at the approximate position (and scale) of the actual clustered HETs. These photos image a section of the thruster exit planes (i.e., looking directly into the annular discharge channels), which is perpendicular to the contour map. It should be noted that to enable various signal processing steps (and prevent information loss), all signals are digitally resampled to a common $2 \mathrm{MHz}$ rate. The colorbar on the right of Figure 5 corresponds only to the $31-\mathrm{cm}$ by 50 $\mathrm{cm}$ plume region electron density contours. The discharge current and photo intensity $\left(F_{T 3}\right.$ and $\left.F_{T 2}\right)$ signals, are mutually in-phase for each thruster, and since a mode- 0 type transient oscillation is occurring, the thrusters are in-phase with each other. During a breathing mode ionization burst, a large quantity of leakage electrons are pushed to the anode, very rapidly producing a large (positive) spike in discharge current while simultaneously the increased ionizing (as well as cross-field electron transporting) collisions emit lots of photons (from atomic state transitions). The rapid electron and photon velocities (compared to the slow ions) serve to place the current and photo signals in phase (with the expected small phase difference being too small to resolve with the finite DAQ rates). The set of six frames in Figure 5 depict a typical breathing mode oscillation, with similar transient behavior repeating indefinitely at a rate of $19.6 \mathrm{kHz}$. Longer sequences confirm the finding in Section D, with $43.4 \%$ of the breathing mode cycles occurring with both thrusters emitting waves of ions in phase with each other.

By considering the fixed radial $(\mathrm{R}=0)$ position on the cluster centerline, axial waves of electron density are observed in Figure 6. The rear wall of this figure contains the temporally synchronous discharge current and photo intensity signals. FASTCAM photos at the corresponding times are also included, and as the rear wall axial coordinate is $0 \mathrm{~cm}$, these photos are coplanar with the thruster exit planes. In this plot, the first oscillations by each thruster are nearly opposite-phase or mode- 1 type. The resulting axial density wave from this mode- 1 event is less distinct since the transient density contributions from each thruster somewhat cancel each other out. The sum of both clustered thruster discharge currents is included (as was done in Figure 2) to show how the density waves track this net signal as anticipated from super-positioning. ${ }^{7}$

\section{Conclusions}

The discharge from two simultaneously operated, low-power Hall thrusters has been temporally and spatially resolved revealing large magnitude property oscillations. These breathing mode oscillations occur in all measured plasma and thruster properties including: thruster discharge currents, electron density, electron temperature, plasma 
potential, and photo intensity. Even with independent thruster setups, a $43.4 \%$ tendency exists for in-phase thruster (mode-0) oscillation. Super-positioning appears to hold with clustered Hall thruster density fluctuations as well as with DC densities. The overall dynamic behavior of clustered Hall thrusters is quite similar to single thruster operation, with both configurations having non-dimensional localized electron density fluctuation magnitudes near $72 \% \pm 10 \%$ throughout the entire downstream near- and far-field plume.

\section{References}

$1 \quad$ Lobbia, R. B., and Gallimore, A. D., A Method of Measuring Transient Plume Properties, AIAA-2008-4650, 44 ${ }^{\text {th }}$ AIAA/ASME/SAE/ASEE Joint Propulsion Conference and Exhibit, Hartford, CT, July 21-23, 2008.

2 Lobbia, R. B., Liu, T. M., Gallimore, A. D., Correlating Time-Resolved Optical and Langmuir Probe Measurements of Hall Thruster Dynamics, SPS-III-36, Presented at the $6^{\text {th }}$ Modeling and Simulation $/ 4^{\text {th }}$ Liquid Propulsion $/ 3^{\text {rd }}$ Spacecraft Propulsion Joint Subcommittee JANNAF Meeting, Orlando, FL, December 8-12, 2008.

3 Choueiri, E. Y., Plasma oscillations in Hall thrusters, Physics of Plasmas, vol. 8, pp. 1411-1426, 2001.

4 Fife, J. M., Martinez-Sanchez, M. and Szabo, J., A Numerical Study of Low-Frequency Discharge Oscillations in Hall Thrusters, 33 ${ }^{\text {rd }}$ AIAA/ASME/SAE/ASEE Joint Propulsion Conference \& Exhibit, Seattle, WA, 6-9 July 1997.

5 Yamamoto, N., Komurasaki, K. and Arakawa, Y., Discharge Current Oscillation in Hall Thrusters, Journal of Propulsion and Power, Vol. 21, no. 5, pp. 870-876, 2005.

6 Hargus, W. and Reed, G., The Air Force Clustered Hall Thruster Program, 38th AIAA/ASME/SAE/ASEE Joint Propulsion Conference and Exhibit, Indianapolis, IN, 7-10 July, 2002.

Beal, B. E., Clustering of Hall Effect Thrusters for High-Power Electric Propulsion Applications, Ph.D. Thesis, University of Michigan, 2004.

8 Beal, B. E., Gallimore, A. D., Haas, J. M. and Hargus Jr, W. A., Plasma Properties in the Plume of a Hall Thruster Cluster, Journal of Propulsion and Power, Vol. 20, no. 6, pp. 985-991, 2004.

9 Beiting, E., Garrett, M., Pollard, J., Pezet, B. and Gouvernayre, P., Spectral Characteristics of Radiated Emission from SPT-100 Hall Thrusters, IEPC-2005-221, $29^{\text {th }}$ International Electric Propulsion Conference, Princeton University, New Jersey, 2005.

10 Hargus Jr, W. and Pote, B., Examination of a Hall Thruster Start Transient, AIAA-2002-3956, 38 AIAA/ASME/SAE/ASEE Joint Propulsion Conference and Exhibit, Indianapolis, IN, 7-10 July, 2002.

${ }_{11} \quad$ Lobbia, R. B., Gallimore, A. D., Fusing Spatially and Temporally Separated Single-point Turbulent Plasma Flow Measurements into Two-dimensional Time-resolved Visualizations, Presented at 12th International Conference on Information Fusion, Paper No. 243, Seattle, WA, July 6-9, 2009. 